\title{
Creative Idea Generation: How To Incorporate Plussing Into Your Creative Thinking Sessions
}

\author{
Whitney Godwin \\ West Virginia University
}

Follow this and additional works at: https://researchrepository.wvu.edu/etd

\section{Recommended Citation}

Godwin, Whitney, "Creative Idea Generation: How To Incorporate Plussing Into Your Creative Thinking Sessions" (2014). Graduate Theses, Dissertations, and Problem Reports. 299.

https://researchrepository.wvu.edu/etd/299

This Thesis is protected by copyright and/or related rights. It has been brought to you by the The Research Repository @ WVU with permission from the rights-holder(s). You are free to use this Thesis in any way that is permitted by the copyright and related rights legislation that applies to your use. For other uses you must obtain permission from the rights-holder(s) directly, unless additional rights are indicated by a Creative Commons license in the record and/ or on the work itself. This Thesis has been accepted for inclusion in WVU Graduate Theses, Dissertations, and Problem Reports collection by an authorized administrator of The Research Repository @ WVU. For more information, please contact researchrepository@mail.wvu.edu. 


\section{Creative Idea Generation: \\ How To Incorporate Plussing Into Your Creative Thinking Sessions}

\section{Whitney Godwin}

Thesis submitted to the P.I. Reed School of Journalism at West Virginia University in partial fulfillment of the requirements for the degree of

Master of Science in Journalism

John Temple, MFA, Chair

Gina Dahlia, MSJ

Emily Corio, MSJ

Anna Elfenbein, Ph.D.

Department of Journalism

Morgantown, West Virginia

2014

Keywords: Plussing, Brainstorming, Creative Thinking, Idea-Generation

Copyright 2014: Whitney Godwin 


\section{Abstract \\ Creative Idea Generation: How To Incorporate Plussing Into Your Creative Thinking Sessions}

\section{Whitney Godwin}

The goal of this master's thesis is to evaluate Disney's "plussing" as a form of idea-generation for every type of organization and business. This method was assessed through a case study of two nonprofit organizations in Morgantown, West Virginia after developing three original plussing methods. Both organizations participated in plussing sessions. Sessions were followed up with a survey of each participant. Findings suggest "plussing" has proven more beneficial to the organizations than traditional brainstorming methods. Several participants stated that plussing provides a more effective way for organizations to produce the best idea and product possible without the fear of criticism from other group members. The information gathered in this study can add to the knowledge of how plussing works, and which organizations find it the most helpful. 


\section{Table of Contents}

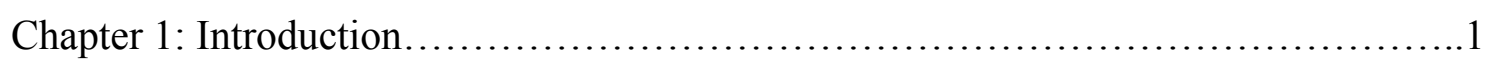

Chapter 2: Literature Review..............................................6

Disney, Pixar, and Plussing............................................. 6

The Merits of Plussing..................................................

Plussing In Practice................................................. 10

Theoretical Framework...............................................11

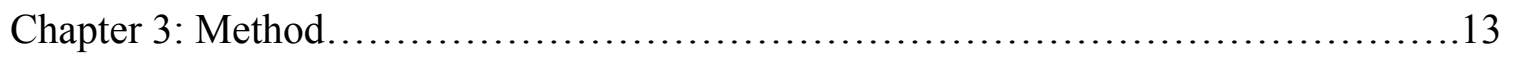

Plussing Method One................................................... 13

Plussing Method Two................................................14

Plussing Method Three...,.............................................. 15

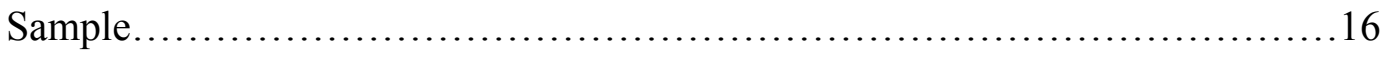

Coding Scheme...................................................17

Strengths and Limitations........................................... 18

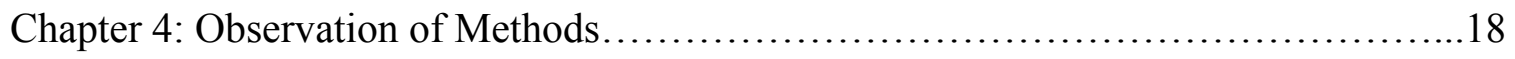

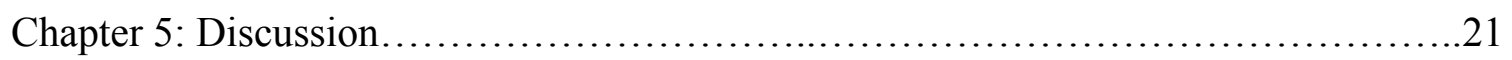

Chapter 6: Conclusions and Future Research....................................23

Chapter 7: Appendices...................................................25

A- Post Plussing Survey..........................................25

B- Participant 1 Survey..............................................27

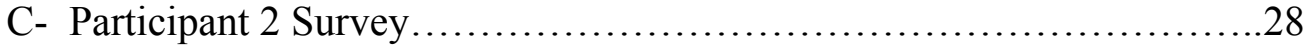

D- Participant 3 Survey...........................................29

E- Participant 4 Survey............................................. 30

F- Participant 5 Survey........................................... 31

References................................................................... 


\section{$\underline{\text { Introduction }}$}

Throughout history, humans have been gathering together to discuss ideas and decisions as a group. Leaders of these groups seek to maximize creativity while minimizing the occurrence of problems that means they must try to tackle the problems head on (Llopis, 2013). One method often used to provoke idea generation is "brainstorming." In 1957, Alex Osborn invented the concept that (Dufree, 2007) Webster's Dictionary defines as “a conference technique by which a group attempts to find a solution for a specific problem by amassing all the ideas spontaneously contributed by its members." According to Dufree (2007), Osborn created three basic brainstorming rules-1) Generate many solutions, 2) Encourage wild ideas, and 3) Defer ALL judgment until later. Osborn believed that the effectiveness of brainstorming could be attributed to both cognitive and social processes (Dugosh and Paulus 2004). As the idea has evolved, some of the basic concepts have stayed the same while others are changing. Numerous studies have been done since Osborn's original research that may prove some cognitive and social processes can hinder creative thinking (Taylor, Barry, and Block, 1958, Paulus 2007, and Dugosh and Paulus, 2004, and Nijstad and Stroebe 2006).

While brainstorming is still popular in many organizations today, creative thinking is starting to challenge some of the concepts of traditional brainstorming. When managers cannot draw from experience or established solutions, they must find solutions in a different way. The more unusual a problem, the more the situation calls for a creative solution (Reckhenrich, Kupp, and Anderson, 2009). According to Jonah Lehrer (2012), even though we are surrounded by human creations, "there is something profoundly mysterious about the creative process." Some scholars (Paulus and Brown, 2007 and Paulus and Yang 2000) feel brainstorming performance hinders creative thinking. Paulus (2007) says a lack of creative thinking may be due to outside 
factors such as social and cognitive influences. Idea generation itself is a cognitive process involving the "retrieval of relevant knowledge from long-term memory and the integration of knowledge in working memory" (Paulus and Brown, 2007). Idea generation involves both the retrieval of existing knowledge and combining experience with other various existing knowledge (Paulus and Brown, 2007 and Dugosh and Paulus, 2004). Working in a group also allows for stimulation of ideas from other group members who may mention a key word that prompts an existing knowledge or idea from another group member. In this aspect, group brainstorming is very helpful to the creative process. Dugosh and Paulus (2004) mention that exposure to ideas from others can stimulate the creative thinking process and lead to the generation of other ideas. However, some scholars (Dugosh and Paulus, 2004, Paulus and Brown, 2007, and Taylor, Barry, and Black 1958) believe group brainstorming can hinder the idea generation process. According to Dugosh and Paulus (2004), several of Osborn's procedural factors have been identified as potential causes for a productivity gap. Factors such as social loafing and freeriding, production blocking, and downward performance matching can all cause ineffectiveness and lack of production when brainstorming (Dugosh and Paulus, 2004). Paulus and Brown (2007) suggest that exposure to other ideas may have inhibiting effects on idea generation. Nijstad and Strobe (2006) argue that group members ideas may interfere with the train of thought of other group members causing them to forget their idea and produce fewer ideas overall. Michael Diehl and Wolfgang Stroebe (1987) say that verbalizing ideas as they occur may cause participants to forget or suppress ideas because they seem less relevant or less original later in the discussion. Rickards (1999) says Osborn's intention was to restructure meetings to overcome things that block idea generation. Osborn also intended for benefits of brainstorming to extend beyond idea generation to team building and higher creative problem solving (Rickards, 1999). 
According to Rickards (1999), Osborn hoped to create a meeting environment that would neutralize social blocks and free up idea generation.

People's perceptions of themselves and what they have to offer to others are a core part of identity (Moeller and Marsh, 2013). In his brainstorming paradigm, Osborn (1954) expressed his concern that some group members may participate less and generate fewer ideas because they are afraid they will be judged by other members of the group — often coworkers and friends (Paulus and Brown, 2007, Taylor, Barry, and Block 1958, Diehl and Wolfgang, 1987). According to Paulus and Brown (2007), this may be true. Groups of people bring a variety of knowledge and experience to any idea generation session. People with more knowledge or expertise on a subject will likely share more ideas causing other members to tune out the discussion or feel inferior to their group members (Paulus and Brown, 2007, and Taylor, Barry, and Black 1958). Aware of this, Osborn decided to create a brainstorming rule that proposed groups should make no positive or negative comments until the group has finished generating ideas before selection (Osborn, 1956). Dugosh and Paulus (2004) agree that conformity pressures and the fear of judgment can hinder creativity.

The most effective leaders approach problems through a lens of opportunity (Llopis, 2013). Teams must be creative enough to recognize that all problems are the same, they're just packaged differently (Llopis, 2013). Creativity is a key skill for organizations in crucial situations such as adapting to change and shaping the market. Creative thinking can actually keep an organization ahead (Reckhenrich, Kupp, and Anderson, 1987).

Is there a type of group idea generation that does not inhibit creativity? Yes, it's called plussing. Plussing is a much more abstract form of creative thinking. Jonathan Lehrer (2012) takes a look at plussing in his book, "Imagine: How Creativity Works." He defines plussing as a 
"technique that allows people to improve ideas without using harsh or judgmental language" (Lehrer, p. 163). While it includes some main creative thinking concepts, plussing is unique for a few reasons. In Osborn's brainstorming paradigm, members of the small brainstorming group vote on the best idea. With plussing, there is no one idea better than another. Each idea is used to create a better overall idea. So the goal of plussing is that when an idea is criticized, the criticism should contain a plus - a new idea that builds on the flaws of the original idea in a productive manner (Lehrer, 2012).

So where did plussing come from? Plussing has been around for years simply because it's often a product of personality. However, the credit for plussing goes to Walt Disney and Pixar animation studios. Pixar suggests the term plussing means "finding what's good about an idea and making it even better." The studio uses several techniques when working on a creative project with its staff. First, group leaders, ask questions such as "What is your project vision?" and "What inspired you to pick this project?" In addition to several other questions, Pixar then asks their employees to evaluate their idea and take note of what the hard parts and the easy parts will be. Following that, the creative thinker in charge will then use other ideas as well as his or her own ideas to build off of the original idea submission.

While the idea of not giving positive or negative feedback applies, plussing involves no selection stage. Instead of choosing one idea over all the others, each idea is important to achieve the best project result possible (Pixar, 2012). Constructive criticism is an important part of plussing because the criticism leads to more important new ideas (Lehrer, 2012). The reason criticism leads to more new ideas is that it encourages us to fully engage with the work of others (Lehrer, 2012). We think about other people's concepts because we want to improve them. In plussing, participants are asked to look at the ideas of the other participants and add a 
construction criticism and possible solution; this means they "plus" the idea adding to it. Lehrer (2012) says it's the imperfections that lead us to really listen to the ideas of others. When everyone is right and every idea is equally useful from the start, then there's no incentive to think about more possibilities or listen to someone else's thoughts and ideas; essentially the problem will remain impossible because the absence of criticism keeps us in the same place (Lehrer, 2012).

Paulus and Brown (2007) also make the argument that in the right situation, this diversity of knowledge can help the process rather than hinder it. Sarah Harvey (2013) says groups whose members have diverse knowledge categories can produce more ideas. Additionally, Havey (2013) also suggests that groups with members from different ethnic backgrounds produce higher quality ideas. Creative output happens in two ways: convergent and divergent thinking (Harvey, 2013). Convergent thinking builds on ideas while divergent thinking combines ideas (Harvey, 2013). This is essentially plussing. In a plussing session, for example, diversity of knowledge can allow other group members to tap into all of the knowledge to produce one larger, better idea by combining everyone's knowledge and experience together. This is ultimately the goal of plussing — to produce the best product possible by using a number of good ideas. An important part of Pixar plussing is that team members are made up of people from different backgrounds. Lehrer (2012) talks about how a creative team for a movie is not completely made up of animators. While animators are an important part of the creative team, computer graphic specialists, technical specialists, and the like are also on the creative teams (Lehrer 2012). Traditionally, many researchers say creative teams should be composed of members with different knowledge and experiences (Harvey, 2013). Specifically, diverse groups of people have an advantage because, “ideas can result when one group member's idea 
stimulates a novel connection in another's associative hierarchy" (Harvey, p. 822). One of the interesting things Pixar does is locate everyone on the creative team in the same area. Whether members of the creative team are engineers, animators, or computer graphic designers, they all coexist in the same area of the Pixar studio (Lehrer 2012). Pixar executives say this is because interactions with outsiders often spark interesting conversation about the creative project when team members least expect it. By widening the knowledge pool, more associations and connections can be made and thus, more ideas can be generated.

\section{Disney, Pixar, and Plussing}

What began as a $\$ 400$ business adventure in 1923 has evolved into a multi-billion dollar corporation. Mickey Mouse made his appearance in an animated short in 1928, known as "Steamboat Willie," and the Walt Disney Company took off. Today, Disney is made up of many facets including film, theme parks, merchandise, musicals and more, but animation remains one of the key staples to the business. Forty-nine video releases have grossed over $\$ 100$ million each, domestically, making Disney's Buena Vista Pictures the all-time industry leader. Seven out of the top 10 selling videos in the world are Disney animations. Aladdin, Tarzan, Beauty and the Beast, Pocahontas, and the Lion King alone have grossed over one billion dollars (Artz, 2004, p.117). In addition, Disney has won over 85 Academy Awards for its animated films through the years. In fact, many scholars (Artz, 2004; Ajayi, 2010; Belkhyr, 2012) would argue Disney no longer serves as an entertainment medium but as a producer of culture.

When Disney merged with Pixar, Pixar Animation Studios came in and completely revamped the management process within the studio (Catmull, 2008). Pixar's leaders had discovered specific practices for structuring and operating a creative organization that they felt would greatly benefit Disney. Pixar has a unique track record of success; it's the leading pioneer 
in animation and has never had to buy scripts or movie ideas (Catmull, 2008). The company stresses that the management is not to prevent risk, but to build the capability to recover when risks result in failure (Catmull, 2008).

Pixar began as a computer manufacturer in 1980 run by George Lucas. It was a division within Lucasfilm, a movie production firm (Lehrer, 2012). Pixar hired Ed Catmull and Alvy Ray Smith, two computer scientists to do digital imagery. Both expressed an interest in computer animation, but Lucas refused to let his scientists be filmmakers. The two continued their animation project in secrecy and eventually hired John Lasseter, a Disney animator as a "userinterface designer" (Lehrer, 2012). When Steve Jobs was run out of Apple, he bought into Lucasfilms. He was intrigued by the Pixar Image Computer, a \$135,00 machine capable of generating complex graphic visualizations (Lehrer, 2012). In 1986, Jobs bought the computer division and named it "Pixar." The computers proved too expensive and not enough consumer interest was generated for profit. Therefore, Catmull and Smith turned to animation to create profit for Pixar (Lehrer, 2012).

In 1995, Pixar Animation Studios released its first full-length animated film, Toy Story; since then, they've created eleven feature films. Each of the eleven films Pixar has produced has been a commercial success averaging an international gross of more than $\$ 550$ million per film (Lehrer, 2012). In addition, every Pixar Film has been nominated for Best Animated Feature since the Oscars inaugurated the category in 2001 (Lehrer, 2012). Five Pixar films have captured the award.

What sets Disney and Pixar studios apart from so many other corporations worldwide? According to Artz (2004), the answer lies in "classic" Disney animation, production, and theme. 
Contributing to the success in the animation, production, and theme creation is an idea known as "Plussing."

Pixar implements plussing nearly every single day. Every morning there is a creative meeting called a "crit session" that sometimes lasts until noon (Lehrer, 2012). The company is set apart by the fact that it appreciates every contribution regardless of who the contribution comes from and how it originated (Catmull, 2008). They seem to be one of the only organizations that have mastered this concept (Lehrer, 2012).

\section{The Merits of Plussing}

You can't deny the success of the Disney and Pixar Company, but is plussing really better than brainstorming? Rickards (1999) says evidence to support lack of quality ideas resulting from brainstorming is inconclusive and lacking. If brainstorming is done without regards to certain factors such as social and cognitive influences, then it can be counterproductive (Paulus and Brown, 2007 and Dugosh and Paulus, 2004). Creative thinking must be carefully thought out before members of a team are asked to generate ideas in the first place. While social and cognitive influences may seem minor, studies show that these can have a great effect on the number of ideas generated (Paulus and Brown, 2007, and Dugosh and Paulus, 2004).

The reason brainstorming is so ineffective goes back to the point about necessary criticism. Jonathan Ives, the lead designer at Apple, says the only way to make progress is to be "brutally critical" (Lehrer, 2012). Ives says in order to maximize group creativity you have to encourage a candid discussion of mistakes. Once these mistakes or issues of an idea are brought to light, the team can then begin building on the idea to improve it. "Acceptance of error reduces its cost" (Lehrer, 2012). Lehere says when you are less worried about your contribution being 
perfect, more candid conversation ensues. He says companies only get things right when they talk about what goes wrong. The criticism is not to encourage ideas, but rather to build them. By choosing one idea in a brainstorming session, nothing ever gets built; instead, one plan gets executed, and no one talks about what went wrong before the next brainstorming session occurs (Lehrer, 2012).

Lehrer (2012) says Pixar's method is so effective because while the group engages in a debate, it's a debate that includes the unexpected with innovative ideas due to "relentless dissent" (Lehrer, p.164). Pixar gives its production teams the freedom to think creatively within a broad spectrum. Managers and producers understand that if they didn't allow for some uncertainty, most of the successful Pixar ideas would never emerge (Reckhenrich, Kupp, and Anderson, 2009). Even after group meetings, Pixar employees admit they think about the ideas at the gym, in their cubicle, or on their commute home. The idea always seems to be on the backburner and the innovative breakthrough may happen at any time. In addition, plussing on a certain project is continued daily at Pixar allowing creative teams the chance to continue idea generation and problem solving (Lehrer, 2012).

According to Young Makers (2013), plussing sessions can offer a monthly deadline so that project teams aren't faced with one huge deadline months in the future. More deadlines allow for tweaks in the overall project that may have a huge impact on the quality of the final product. Plussing sessions give all teams a chance to see the creativity and breadth of ideas of the entire group, and teams can see other projects develop through the season. Plussing sessions also bring more positivity. Since every idea is incorporated in plussing sessions, these sessions give project teams the chance to discuss their failures in constructive ways. Additionally, plussing sessions provide an opportunity to build relationships and a trusting community between project 
members. By always looking at the positives and improving projects, plussing can help build a positive community that is focused on helping one another and the company achieve more (Young Makers, 2013).

\section{Plussing in Practice}

Plussing isn't always flawless. Lehrer (2012) notes that even plussing can't prevent the occasional heated argument or hurt feelings. Pixar also openly admits that the process requires many grueling hours that fall outside of the typical demands of a 9am-5pm workday. Lehrer (2012) also reminds readers that plussing will not always produce magical ideas every day.

The risk to plussing is that if you want to be original and creative, you have to accept the uncertainty that comes along with it, and be able to recover when your organization takes a risk and fails (Catmull, 2008). Additionally, plussing will require more time than an hour long brainstorming session. Plussing is a creative processs. The process is not limited to an hour. As previously mentioned, the plussing process continues through the entire life of the project until it's published and distributed. Time is what must be exchanged for creativity, originality, and success.

The production of Toy Story 2 shows Pixar how valuable plussing was to its organization despite the time demand. According to Catmull, Pixar executives felt that Toy Story 2 lacked a certain spark that every other Pixar film had. This is when Pixar decided to move everyone working on the film, engineers, animators, storywriters, and everyone else involved in the creation of the film, to the same set of cubicles. Pixar knows the best ideas often come from the most random conversations because interactions prompt you to think about something you may not have given much thought to before (Catmull, 2008). According to Catmull (2008), there were so many mistakes they did not expect the production to be done on time. In fact, Pixar, stopped 
production on almost every other film to concentrate its efforts solely on Toy Story 2. As a result of never giving up on plussing and hashing out the mistakes and issues, Toy Story 2 was released on time and became on of the company's most successful films (Catmull, 2008). Catmull admits there were a lot of long hours put in, and several people even suffered health issues due to stress (Catmull, 2008). Catmull (2008) explained the most important thing Pixar learned from the movie is that talent isn't enough. "Talent fails every day. We want to screw up and fix it together. Knowing your first idea isn't going to be the best idea is a huge part of plussing, that's why plussing works" (Catmull, 2008).

\section{Theoretical Framework}

Many scholars believe social-motivational factors affect idea generation regardless of the technique used for creative thinking (Paulus and Brown, 2007 and Moeller and Marsh, 2013). Leon Festinger's social comparison theory suggests that people will be conscious about what they say in group. The theory says social comparisons occur when one person relates their ability and opinions to those of other people in the group (Moeller and Marsh, 2013). At the same time, some scholars (Paulus and Brown, 2007 and Moeller and Marsh, 2013) argue that comparing oneself to other group members drives competition and leads to higher performance overall.

Another theory that supports the flaws of brainstorming is the economic theory of good (Diehl and Stroebe, 1987). This theory states that the temptation to free ride varies with group size because it decreases the perceived effectiveness of individual contributions as well as lowers the identifiabilty of individual contributions (Diehl and Stroebe, 1987). Thus, anything that reduces the ability of the experimenter to monitor individual productivity is likely to reduce the motivation to work. Group members expect their ideas to be pooled and evaluated at the group 
level so they are tempted to free ride off of others. People know they will be monitored individually are more likely to be more productive and efficient (Diehl and Stroebe, 1987).

So how exactly does plussing work? That's the million-dollar question. While many authors and websites provide insight on how to possibly combine a plussing session and creative thinking, little research has been done to explore steps for plussing and how to use plussing effectively. Catmull suggested a few things companies can do to ensure that plussing is successful without ever divulging a specific method.

First, plussing will not work unless group leaders empower group members by giving the creative team control over every stage of idea development (Catmull, 2008). Second, for plussing to be successful, there must be a peer culture that encourages everyone to help each other produce their best work possible (Catmull, 2008). Third, it's important to make sure communication happens freely. The most important way to solve complext problems is to trust people to address problems without getting permission from a supervisor (Catmull, 2008). Finally, crafting a learning environment is one of the most important parts of creative thinking. Reinforcing that everyone is learning by requiring everyone to participate and plus one another helps ensure that people feel comfortable sharing every idea is key (Catmull, 2008). By inviting everyone to use constructive criticism with every single idea, the idea of being the only one criticized is taken away. Everyone's idea will be critiqued, plussed, and improved putting everyone in the same category.

Creativity is crucial when it comes to plussing. Reckhenrich, Kupp, and Anderson (1987) explain that there are three core concepts of creative thinking starting with personal creativity and expanding to being creative in a social context. The first core concept of creative thinking is active personal creativity that comes from within (Reckhenrich, Kupp, and Anderson, 1987). The 
second concept, the sculptural theory, is creativity during the process. The third concept is a social sculpture theory that involves creativity collaboratively. Using an element of each of these creative thinking core concepts, this study will seek to create three concrete plussing methods.

This study seeks to create three different methods of plussing and how local nonprofit organizations can use for their creative projects. Specifically this study will seek to answer find an effective method to execute plussing by testing three original plussing methods I created. The study also seeks to answer if plussing is a more productive approach for idea generation at nonprofit organizations than traditional methods of brainstorming. During the course of the study, body language will be observed as well as if plussing lessens the tendency to criticize people of different social status or minority.

\section{Methodology}

For the purposes of this study, I created three original plussing methods that were used during idea-generation sessions, at two separate nonprofit organizations. Each process is structured, but not so much that it hinders creative thinking. Each organization will use a different plussing method instead of its usual brainstorming tactics. In each case the board of directors or executive board will hash out an original idea that they would like to implement into their organization. Once the initial idea is created, then a creative team will be asked to build on the idea and essentially make it happen.

\section{Method One - Silent/Written Group Plussing}

- Team members were divided into groups of three.

- Participants spent two minutes writing down their ideas to the problem on their notecard. 
- They passed their cards to the person to the right, and were given two minutes to respond to the idea on the card they received. Instead of changing the idea, they were instructed to add to the idea. This step was then repeated until every card reached every participant.

- After everyone received their notecard back, participants, discussed all of the ideas and how to connect them together into the project. All ideas and plusses mentioned in the group discussion were written down.

- Following the group discussion, a post-plussing survey was distributed. (Appendix A).

\section{Method Two- Verbal Team Word Association Idea Generation}

- Team members began generating ideas verbally as a group, rather than individually as in method one.

- Group members were introduced to the topic. They were then instructed to come up with words they associated with the topic. Intuition is an emotional side of creative thinking (Reckhenrich, Kupp, and Anderson, 1987). Word association is a form of intuition. Employees should not be forced to rationalize their decisions.

- Group members were reminded there is no correct answer and to think outside of the box.

- Group members were given the word or topic of discussion, and given 15 minutes to think of words associated with the topic of discussion.

- Words associated with the idea were placed on a white board for all group members to see.

- The group discussed how these words can be connected to the idea. Team members were encouraged to bounce ideas off of one another about the final project. 
- A candid discussion of possible mistakes in ideas was encouraged. However, in order for group members to criticize an idea, they were instructed to plus it and add a productive suggestion as a solution to that specific idea.

- Following the discussion, a survey was distributed on plussing method 2 (Appendix A).

\section{Method Three-Oral/Written Group Idea Generation}

- Method three was an oral group idea generation process. Like method two, the members of the creative team were given the topic of the creative project.

- Ideas were bounced around from all group members and put on the whiteboard for all to see. Group members wrote down ideas or suggestions during discussion.

- After an original list of ideas was generated, and the participants addressed each idea individually and were asked to add to that idea. After each idea generated multiple sub-ideas, the group was asked to make connections between the ideas presented on the board.

- After making connections as a group, individual team members were asked to free write about the discussion, generate ideas, and any connections made. Team members were given a chance to review everyone's free write, and a final group discussion took place.

- As always in any plussing method, debate and constructive criticism were encouraged if the participant added a plus to the idea rather than simply rejecting it.

- The group member criticizing the idea should always explain why they don't feel the idea will work and then build on it (Lehrer, 2012). After this discussion, a survey on plussing method three will be distributed.

- Following the final plussing session, surveys about the entire experience were given to participants to fill out (Appendix A). I then collected the surveys and began analyzing data. 


\section{Sample}

Since plussing is a form of idea generation, it can be implemented in any organization, whether it's a for-profit organization, a not-for-profit organization, a news organization, an educational institution, or a government institution. Therefore, for the purpose of this study, two local nonprofit organizations were the sampling frame. Each of the three plussing methods were implemented at each nonprofit organization.

The first nonprofit that participated in the plussing activity was Stepping Stones.

Stepping Stones is a nonprofit recreational organization for people with disabilities, and is based in Morgantown, West Virginia. It is the only organization of its kind in the nation (Stepping Stones, 2013). Stepping Stones goal is to, "help people achieve their maximum level of independence through innovative recreational activities" (Stepping Stones, 2013). Stepping Stones boasts a state-of-the-art facility that offers amazing year- round programs such as soccer, basketball, wheelchair basketball, summer/day camp, golf, computer classes, therapeutic riding, social activities, and a fully accessible swimming pool as well as other social activities such as dances and pageants.

Plussing is a good idea generation technique to implement for Stepping Stones because they're always looking for new ways to get their participants involved. In addition, according to recreation coordinator Kim Walls, the organization is looking to revamp its website in the near future. A creative plussing session could greatly benefit the organization on how to move forward with these projects.

The second nonprofit organization that explored plussing was Crossroads Church of Westover, West Virginia. Crossroads Church is a member of the Acts 29 Network of churches and, "exists to spread a passion for Jesus Christ for the joy of all people within the city and 
extending to all nations" (wvcrossroads.com/who-we-are.php). The organization recently added a video and creative team to improve the organization.

A creative plussing session benefited the organization by allowing the creative projects and videos to push the envelope and provide the best possible product to the church and the community. Especially for lengthier videos, plussing is a great activity to help ensure that the videos promote the vision and passion of the church and their events. Plussing can also be applied to more areas of the church other than creative team and video team. It would be very beneficial to other areas of the church as well such as hospitality and community outreach.

These two very different nonprofit organizations will help show how plussing can be applied in literally any idea generation setting. With a variety of plussing methods, there can be a way for almost every organization to implement plussing and use it effectively for the benefit of their company or organization.

\section{Coding Scheme}

In these case studies, I implemented each plussing method in each of the two nonprofits, then issued a survey to participants afterward. The simple survey conveys the opinion of the participants in the study on the concept of plussing. In order to construct a comprehensive tool for investigation, I used the following coding scheme:

Brainstorming. Brainstorming is a conference technique by which a group attempts to find a solution for a specific problem by amassing all the ideas spontaneously contributed by its members" (Dufree, 2007).

Creative Thinking. As previously mentioned, is a brainstorming process that uses a different way of looking at problems or situations from a new perspective rather than using only brainstorming techniques (Lehrer, 2012, and Paulus and Brown, 2007). 
Group Brainstorming. Group brainstorming, as mentioned previously, is working with one or more people to generate ideas. According to Dugosh and Paulus (2004) stimulation of ideas from others can stimulate creativity.

Idea Generation. Idea generation is the, "retrieval of relevant knowledge from long-term memory and the integration of knowledge in working memory" (Paulus and Brown, 2007)

Plussing. Jonah Lehrer (2012) defines plussing as, "a technique that allows people to improve ideas without using harsh or judgment language"

Surveys from each participant were read and analyzed when considering the success of the study. Surveys were also used to determine which plussing method the majority of participants preferred.

\section{$\underline{\text { Strengths and Limitations }}$}

One strength of this case study is the fact that it's one of the first of its kind. Many scholars have written numerous articles about brainstorming and creative thinking, but little research exists on plussing. This study will at least add a little clarification on the topic of plussing and how it can be used effectively. This study will help nail down a specific plussing method. On the other hand, the lack of plussing research could be viewed as a limitation. Without much research the concept of plussing is abstract and broad. In addition, due to time constraints, only two nonprofit organizations could be tested.

\section{Observation of Methods}

Stepping Stones was the first organization I observed. The organization was brainstorming for its annual art festival event held in April. A team of three people was engaged with this project: the administrative assistant, the executive director, and the recreation coordinator. They generated ideas for everything from food for the event to entertainment 
choices. Originally, I had planned to visit the organization three times, however, Stepping Stones, does not hold weekly meetings. As a small nonprofit organization, they only hold meetings when necessary for events. Therefore, I had to implement all three plussing methods in the same meeting over the course of two hours. Before each method, I set up a camera to be able to capture body language and any observations I may have missed in person.

First, I observed them as they used their traditional brainstorming tactic for the event. After about thirty minutes, I asked them to use method three, oral group idea-generation, because it was closely related to the method they were already using. They were taking notes as they discussed their project. They used method three to discuss things like art activities for the events.

Second, we flowed from method three into method two. They still used the same paper to write down words that could be related to the event and generate ideas using the words for the entertainment and demonstrations portions of their event.

Next, I gave each of them a note card and we went into method one. I asked each of them to write down what they needed to improve about the event and how they could do so. Then after three minutes, I had them pass their card to the right, and plus the idea on the note card. They repeated this process every three minutes until everyone had the chance to plus each card.

Finally, there was a group discussion about the cards and the notes made. The discussion was helpful, and even more ideas were generated during discussion post-plussing. After the group discussion, participants filled out the post-plussing survey about the methods they used.

The second group, Crossroads Church, has several groups that meet sporadically throughout the week. Each group is unique and focuses on one aspect of the church's needs. The Crossroads Church Creative Team is the group that agreed to participate in the plussing activity. Again, this was a very small group of just two people. 
I met three times with this group to observe their group idea generation and implement the three plussing techniques into its brainstorming process. The first week, I observed the group's current strategy for idea generation. Due to the fact they regularly meet in a public café in Morgantown, I was unable to set up a camera to film; however, I did take notes while observing to record any interesting body language, gestures, etc.

The team is a fairly new one, so for the first meeting, they discussed things they hoped to accomplish as a group at the church. Everything from weekly videos to branding was mentioned. They used a typical brainstorming method where they just bounced potential ideas off of one another and decided on one idea for a couple of the videos.

At the next meeting, plussing began. First, the group used plussing method three. They took notes on their laptops as the meeting went along. As one person posed an idea, the other person would write it down and then add a suggestion to the idea on how to improve it, or what direction to go with it. As there were only two people, this was almost the same method as plussing method one with notecards. Therefore, the group only did the exercise once.

At the same meeting, the group also used plussing method two, the word association activity. They used this method specifically to come up with a creative theme for the church's Easter service. They wrote down words, phrases, and imagery of things that reminded them of the Easter story, and then grouped them together to come up with the theme. They then discussed the theme and how it could be applied and carried out efficiently. At the end of the plussing activities and discussion, they filled out the post-plussing survey with their opinions on the new method. 


\section{$\underline{\text { Discussion }}$}

Both organizations seemed to enjoy the plussing experience and members from both expressed that it was a good experience to use a new idea generation tactic. All participants indicated they had never used plussing. Although, one participant explained on their survey, "I feel like sometimes we use some parts of the plussing tactic without noticing." One participant explained, "I enjoyed it. Plussing allows for the best ideas to come about and be expanded on. It allows every idea to be thrown on the table and everyone to build on top of one another." All five participants from both groups indicated on the survey that they preferred group idea generation. They all also indicated they believe group idea generation leads to more substantial ideas. Additionally, all participants in the study indicated that group idea generation stimulates creative thinking.

Interestingly, all five participants in the study indicated that brainstorming in a group setting does not hinder creative thinking. However, some participants noted that this was dependent upon the group because some team members may be afraid to voice their opinion in a group situation. One participant noted, "If the team isn't comfortable with each other, it's normally just the loudest person whose idea we go with." Additionally, the majority of participants said they felt more comfortable plussing. Even the team of two people hesitated to voice their opinion to one another at the first meeting; it seems the written component of plussing allows people to be honest with one another with less fear of judgment. Therefore, many participants preferred method one of plussing. However, none of the participants expressed negative thoughts toward the other methods of plussing. Some participants even noted that plussing as a whole changed the atmosphere of the meeting. "I see how method one would be useful to reiterate certain points, and to see what everyone took away from the 
meeting individually," explained one participant. Another noted, "It gave me a different view on how we work together, think individually, and how to try new thinking and communication."

The Crossroads Church creative team seemed more willing to apply the plussing activity than the Stepping Stones group. The Stepping Stones group tended to revert back to their original brainstorming method. Although participant surveys indicated that they would be willing to try and implement this technique but for individual topics as opposed to a whole event. This could be because plussing requires a longer amount of time than traditional brainstorming, and plussing was originally created and traditionally is used for creative projects_-primarily Disney/Pixar's animated filmmaking. These movies took years to create, produce, and be completed. Thus, sometimes plussing is a lengthy process that takes a few years to complete successfully. The surveys also indicated that the Crossroads Church creative team preferred the plussing method to their traditional method of idea generation. One participant explained, "I loved it! Such a fresh way to encourage ideas. I like that it stimulates creativity while removing the negative fear of being criticized. The only con may be that, occasionally, for the betterment of the idea. Much like a condemned house, needs to be torn down in order to be rebuilt again." By the end of the sessions, the participants from the Crossroads Church group were actually saying things like, "let's use plussing here." After the last session, they continued to express how much they loved plussing and how they planned to implement it as their regular method of idea-generation for the creative team.

"Does plussing lessen the tendency to not criticize people of different social status or a minority," I found no indication that plussing does lessen the tendency to criticize people based on socio-economic status or minority. Both groups were made up entirely of White Caucasion 
Americans. All were around the same socio-economic status and power position status.

Therefore, it was unclear as to whether plussing lessens the tendency to criticize people of different social status or minority.

After observing both groups using the plussing methods, I didn't necessarily notice any body language that suggested the participants preferred one plussing method to another or plussing over brainstorming. However, I did notice that when I observed them in their natural idea-generation method, some participants were hesitant to speak their opinion when generating

ideas orally. When participants used written methods of idea-generation such as plussing method one, they did not hesitate to write down their ideas and wrote freely.

Overall, this case study suggests that some parts of plussing are similar to traditional brainstorming methods like taking notes during a meeting or a group discussion. Participant responses suggest that plussing is a preferred method, because the written component, especially that of method one, allows one to freely express their idea with less fear of judgment. Through observation and participant surveys, it seems plussing may be better suited for idea generation of creative projects such as video, website and graphic design, themes, etc. as opposed to the planning of an annual event. It seems less likely to yield great results in a day-to-day setting or for a project that is on a short time schedule. This may be due to the fact that plussing takes longer than traditional brainstorming because it allows for more creativity.

\section{Future Research}

This study is extremely important to future research on plussing. As previously mentioned, little research exists as to how plussing works and what exactly it is. Being able to define exactly how plussing is conducted would be very beneficial to people in every professional field. In addition, once a plussing method is established, other researchers can use 
these methods to test the effectiveness of plussing versus brainstorming and creative thinking. Testing the plussing method several times with a larger sample would help determine if one method is more effective than another or if participants prefer one method over another. Several other case studies with various organizations such as this study, should be conducted to help determine if there is an exact plussing method that works best for certain organizations. Additionally, it would be interesting to research whether plussing affects the dynamic between teams with a lot of diversity. A diverse group of people may allow for more productive and creative plussing sessions, or it could be hindered due to various backgrounds. Therefore, it would be interesting to conduct a similar study using a larger sample with a more diverse population. 


\section{Appendix A \\ Post-Plussing Survey}

*All answers to this survey will be kept confidential. You are not required to answer any of the questions, but participation is encouraged and appreciated.

Name of your organization (optional):

Position you hold in your organization (optional):

Name (optional):

1. Have you ever used plussing before today? yes no $\mathrm{n} / \mathrm{a}$

2. Which plussing method did you find most helpful during group idea generation? method 1 method 2 method 3

3. Please explain why you chose the method you preferred.

4. What type of idea generation does your organization currently use?

brainstorming plussing creative thinking Other, please explain:

5. Do you prefer group idea generation or individual idea generation? group idea generation individual idea generation

6. Do you think group idea generation leads to more substantial ideas? yes no $\mathrm{n} / \mathrm{a}$

7. Do you think group idea generation stimulates creative thinking? yes no $\mathrm{n} / \mathrm{a}$ 
8. Do you think group brainstorming in a group setting hinders creative thinking? yes no $\mathrm{n} / \mathrm{a}$

9. Explain your plussing experience from today. Did you like it? Did you find it helpful? Please explain your answers.

10. What are some ways you can think of to improve the plussing methods you used today?

11. What other ways can you think of to help facilitate creative thinking?

12. Is there anything else you would like to say about plussing, creative thinking, or brainstorming? 


\section{Appendix B}

\section{Stepping Stones Participant 1 Survey:}

1) Have you used plussing before today? No.

2) Which plussing method did you find most helpful during idea generation? Method 1.

3) Explain why you chose the method you preferred? One idea leads to another idea

4) What type of idea generation does your organization currently use? Brainstorming and Creative Thinking

5) Do you prefer group idea generation or individual idea generation? Group Idea Generation

6) Do you think group idea generation leads to more substantial ideas? Yes.

7) Do you think group idea generation stimulates creative thinking? Yes.

8) Do you think group brainstorming in a group setting hinders creative thinking? No.

9) Explain your plussing experience from today. Did you like it? Did you find it helpful? Please explain your answers. "Helpful! Causes the next person to get a great thought and it adds to the conversation."

10) What are some ways you can think of to improve the plussing methods you used today? Good as is.

11) What are other ways you can think of to help facilitate creative thinking? Sharing ideas.

12) Is there anything else you would like to say about plussing, creative thinking, or brainstorming? "Work as a team on the project and be open-minded to new thoughts and ideas." 
$\underline{\text { Appendix C }}$

\section{Stepping Stones Participant 2 Survey:}

1) Have you used plussing before today? No.

2) Which plussing method did you find most helpful during idea generation? Method 1 and Method 2.

3) Explain why you chose the method you preferred? Method 2 because we are a small office of four that are very close and honest with each other. I do see how method one would be useful to reiterate certain points and see what everyone takes away from the meeting.

4) What type of idea generation does your organization currently use? Brainstorming and Creative Thinking

5) Do you prefer group idea generation or individual idea generation? Group Idea Generation

6) Do you think group idea generation leads to more substantial ideas? Yes. It depends on the group.

7) Do you think group idea generation stimulates creative thinking? Yes.

8) Do you think group brainstorming in a group setting hinders creative thinking? No, but it depends on the group.

9) Explain your plussing experience from today. Did you like it? Did you find it helpful? Please explain your answers. "Yes, I liked it. It gave me a different view on how we work together and ideas of how to try new thinking and communicating."

10) What are some ways you can think of to improve the plussing methods you used today? None.

11) What are other ways you can think of to help facilitate creative thinking? Encouraging communication

12) Is there anything else you would like to say about plussing, creative thinking, or brainstorming? "I liked the experience of plussing." 


\section{Stepping Stones Participant 3 Survey:}

1) Have you used plussing before today? No.

2) Which plussing method did you find most helpful during idea generation? Method 1 and Method 2.

3) Explain why you chose the method you preferred? "I really like the teamwork. Sometimes while talking through an idea we realize it's really good or not so good."

4) What type of idea generation does your organization currently use? Brainstorming and Creative Thinking

5) Do you prefer group idea generation or individual idea generation? Group Idea Generation

6) Do you think group idea generation leads to more substantial ideas? Yes.

7) Do you think group idea generation stimulates creative thinking? Yes.

8) Do you think group brainstorming in a group setting hinders creative thinking? No.

9) Explain your plussing experience from today. Did you like it? Did you find it helpful? Please explain your answers. "I enjoyed the plussing exercise. It gave us the opportunity to speak openly without hurting other people's feelings."

10) What are some ways you can think of to improve the plussing methods you used today? Limiting it to one topic at a time rather than for the whole event.

11) What are other ways you can think of to help facilitate creative thinking? Using it for multiple different events like fundraising and new programs.

12) Is there anything else you would like to say about plussing, creative thinking, or brainstorming? It was a good experience and definitely something we will try and utilize in the future with our organization. 


\section{$\underline{\text { Appendix E }}$}

\section{Crossroads Church Participant 1 Survev:}

1) Have you used plussing before today? No.

2) Which plussing method did you find most helpful during idea generation? Method 1 and Method 3.

3) Explain why you chose the method you preferred? I enjoyed it.

4) What type of idea generation does your organization currently use? Brainstorming and Creative Thinking

5) Do you prefer group idea generation or individual idea generation? Group Idea Generation

6) Do you think group idea generation leads to more substantial ideas? Yes.

7) Do you think group idea generation stimulates creative thinking? Yes.

8) Do you think group brainstorming in a group setting hinders creative thinking? No

9) Explain your plussing experience from today. Did you like it? Did you find it helpful? Please explain your answers. "Yes, using a new idea generator method within a group will always produce more creativity."

10) What are some ways you can think of to improve the plussing methods you used today? No Answer.

11) What are other ways you can think of to help facilitate creative thinking? I believe it was shown today with our clear explanation of what plussing is, the group can get behind it and work with it, and improve ideas.

12) Is there anything else you would like to say about plussing, creative thinking, or brainstorming? No Answer. 


\section{$\underline{\text { Appendix F }}$}

\section{Crossroads Church Participant 2 Survey:}

1) Have you used plussing before today? No.

2) Which plussing method did you find most helpful during idea generation? Method 1

3) Explain why you chose the method you preferred? It stimulates creativity and creates a positive atmosphere.

4) What type of idea generation does your organization currently use? Brainstorming and Creative Thinking

5) Do you prefer group idea generation or individual idea generation? Group Idea Generation

6) Do you think group idea generation leads to more substantial ideas? Yes.

7) Do you think group idea generation stimulates creative thinking? Yes.

8) Do you think group brainstorming in a group setting hinders creative thinking? No

9) Explain your plussing experience from today. Did you like it? Did you find it helpful? Please explain your answers. "I loved it! Such a fresh way to encourage ideas. I like that it stimulates creativity while removing the negative fear of being criticized.

The only drawback may be that occasionally something negative may need to be said for the betterment of the idea. Much like a condemned house needs to be torn down, in order to be rebuilt again."

10) What are some ways you can think of to improve the plussing methods you used today? No Answer.

11) What are other ways you can think of to help facilitate creative thinking? Group bonding projects, listening to music, exposing ourselves to good art.

12) Is there anything else you would like to say about plussing, creative thinking, or brainstorming? I LOVE PLUSSING. 


\section{$\underline{\text { Bibliography }}$}

AmericasBestHistory.com (Eds). (2013). America's Best History Timeline. Retrieved from: http://americasbesthistory.com/

Artz, L. (2004). The Righteousness of Self-Centered Royals: The World According to Disney Animation. Sage 18(1), 116-142.

Ajayi, L. (2010). A Multilteracies Pedagogy: Exploring Semiotic Possibilities of a Disney Video in a Third Grade Diverse Classroom. Springer: Urban Review. 43(1), 396- 413.

Bandura, A. and McClelland, D.C. (1977). Social Learning Theory. New York: General Learning Press.

Belkhyr, S. (2012) Disney Animation: Global Diffusion and Local Appropriation of Culture. International Journal of Human Sciences. 9(2), 704-712.

Burrows, P. and Grover, R. (2006) Steve Job's Magic Kingdom. Business Week. (3970), 62-64.

Casmir, F. and Packman, H. (1999) Learning from the Euro Disney Experience: A Case Study in Internationa1/Intercultural Communication. International Communication Gazette 61(6), 473-489.

Clements, R. (Producer) \& Musker, J. (Director). (1992). Aladdin [Motion Picture] United States: Disney.

Croce, P. (1991). A Clean And Separate Space: Walt Disney in Person And Production. Journal of Popular Culture. 25(3), 91-105.

Connelly, J. and Connelly, M (2011) History of Animation. TechTrends. 55(3), 6-7

Disney Enterprises. (Eds) (1997) Disney's Treasury of Children's Classics: From Snow White and The Seven Dwarfs to Pocahontas. New York: Welcome Enterprises Inc. 
Disney Movies List. (February 2013). Accessible:

http://www.disneymovieslist.com/disney-movies.asp

Do Rozario, R. (2004) The Princess and the Magic Kingdom: Beyond Nostalgia, the Function of the Disney Princess. Women's Studies in Communication. 27(1), 34- 57.

Dugosh, K. and Paulus, P. (2004). "Cognitive and Social Comparison Processes in Brainstorming.” Journal of Experimental Social Psychology. 41(1), 313-320.

Faherty, V. (2001). "Is the Mouse Sensitive? A Study of Race, Gender, and Social Vulnerability in Disney Animated Films." Studies In Media \& Information Literacy Education. 1(3), 1-8.

Fonda, D., Locke, L., Ressner, J., Corliss, R. (2006). “When Woody Met Mickey.” Time 167(6).

Forgacs, D. (1992). Disney Animation and the Business of Childhood. Screen. 33(4), 361-374.

Goldmark, D. and McKnight, U. (2008). Locating America: Revisiting Disney's Lady and the Tramp. Social Identities. 14(1), 101-120.

Gilliam, K and Wooden, S. (2008). Post-Princess Models of Gender: The New Man in Disney/Pixar. Journal of Popular Film and Television. 36(1), 2-8.

Inge, M. T. (2004). Walt Disney's Snow White and The Seven Dwarfs: Art, Adaptation and Ideology. Journal of Popular Film and Television. 32(3).132-142.

Krippendorff, K. (1969). Models of messages: three prototypes. The Analysis of Communication Content. New York: Wiley.

Krippendorff, K. (1980). Content Analysis. An Introduction to its Methodology. Beverly Hills: Sage.

Lehrer, J. (2012). Imagine: How Creativity Works. Houghton Mifflin Harcourt Publishing Company. New York, NY. 
MacIntyre, A. (1998). A Short History of Ethics. Notre Dame: University of Notre Dame.

Mayring, P. (2000). Qualitative Content Analysis. Art. 1(2), 1-8.

Moller, J. and Marsh, H. (2013). "Dimensional Comparison Theory. Psychological Review. 120(3), 544-560.

Nijstad, B. and Stroebe, W. (2006). "How the Group Affects the Mind: A Cognitive Model of Idea Generation: A Cognitive-Social-Motivational Perspective of Brainstorming." Social and Personality Psychology Compass. 1(1): 248-265.

Ohmer, Susan (1993). That Rags to Riches Stuff: Disney's Cinderella and the Cultural Space of Animation. Film History. 5(2) 231-249.

Paulus, B. and Brown, V. (2007). Twoard More Creative and Innovative Group Idea Generation: A Cognitive-Social-Motivational Perspective of Brainstorming. Social and Personality Psychology Compass. 1(1), 248-265

Rickards, T. (1999). "Brainstorming revisited: A question of context.” Oxford, UK.

Springer, C. (1986). Military Defense Department Films from World War II and Vietnam. Cultural Critique. 3, 151-167.

Stapleton, C. and Trowbridge, S. (2009). Melting The Boundaries between Fantasy and Reality. IEEE Computer Society. 42(7), 57-62.

Taylor, D., Berry, P., and Block, C. (1958). “Does Group Participation When Using Brainstorming Facilitate or Inhibit Creative Thinking?" Administrative Science Quarterly.3(1), 23-47.

Telotte, J.P. (2010). The Stereoscopic Mickey: Space Animation, and the Mouse. Journal of Popular Film and Television. 36(3), 133-140. 
Thomas, B. (1976). Walt Disney: An American Original. The Walt Disney Company. New York: NY.

Thomas, F. (1984). Can Classic Disney Animation Be Duplicated on the Computer? Computer Pictures. 41(1), 20-24.

Thomas, F. and Johnston, O. (1981). The Illusion of Life: Disney Animation. Walt Disney Productions. New York: NY.

Thompson, Anne. (2003). Losing Nemo. New York. 36(31), 24-25.

Trifonas, P. (2001). Stimulations of Culture: Disney and the Crafting of American Popular Culture. Educational Researcher. 30(1), 23-28.

Watts, S. (1995). Walt Disney: Art and Politics in the American Century. The Journal of American History. 82(1), 84-110.

Wonderly, M. (2009). Children's Film as an Instrument of Moral Education. Journal of Moral Education. 38(1), 1-15.

YoungMakers. (2012). "Plussing Sessions." Retrieved September 3, 2013 from http://youngmakers.org/plussing-sessions

Zarranz, L. (2007). Diswomen Strike Back? The Evolution of Disney's Femmes in the 1990s. Atenea. 27(2), 55-63. 